\title{
SECURING THE GLOBAL AIRSPACE SYSTEM VIA IDENTITY-BASED SECURITY
}

\author{
William D. Ivancic, NASA Glenn Research Center, Cleveland, Ohio
}

\begin{abstract}
Current telecommunications systems have very good security architectures that include authentication and authorization as well as accounting. These three features enable an edge system to obtain access into a radio communication network, request specific QoS requirements and ensure proper billing for service. Furthermore, the links are secure. Widely used telecommunication technologies are LTE and WiMAX
\end{abstract}

This paper provides a system-level view of network-centric operations for the GAS and the problems and issues with deploying new technologies into the system. The paper then focuses on applying the basic security architectures of commercial telecommunication systems and deployment of federated Authentication, Authorization and Accounting systems to provide a scalable, evolvable reliable and maintainable solution to enable a globally deployable identity-based secure airspace system.

\section{Background}

The air transportation community is continually looking to improve the safety and efficiency of the GAS. This community consists of a variety of national and international government organizations, airlines, and equipment suppliers and manufacturers. In general they have the common goal stated above, but in practice each organization has different stakeholders with often conflicting bottom-line goals. For example: the FAA and EUROCONTROL primary goal is safety of flight, safety of life. While this is also a goal of the airlines, they must meet this goal while remaining profitable. The ICAO and the IATA cooperate to ensure interoperability of the GAS. Groups such as NASA are chartered to develop new technologies directed at improving the safety and efficiency of the GAS.

The FAA is the national aviation authority of the United States. It has authority to regulate and oversee all aspects of American civil aviation. The FAA's mission is to provide the safest, most efficient aerospace system in the world.
EUROCONTROL is the European Organisation for the Safety of Air Navigation. It is an international civil organization working for seamless, pan-European air traffic management. EUROCONTROL coordinates and plans air traffic control for all of Europe.

The ICAO, is an agency of the United Nations. It codifies the principles and techniques of international air navigation and fosters the planning and development of international air transport to ensure safe and orderly growth.

The IATA is a trade association of the world's airlines with membership of roughly 260 airlines. IATA's mission is to represent, lead and serve the airline industry. They work in a number of areas including policy, safety, environmental impact, security and simplifying business to improve efficiencies and save money.

In order to improve efficiencies and maintain safety two major programs have been created - one in the United States and another one in Europe. These are the NextGen in the United States and SESAR. NextGen is an ATC modernization program which uses sophisticated technologies and new flight procedures to reduce flight delays, flight times and aircraft fuel burn/emissions. One of the key technologies is GPS. GPS should enable planes to fly closer together, take more direct routes and avoid delays caused by airport "stacking". SESAR is a collaborative project to completely overhaul European airspace and its air traffic management (ATM).

NASA's AOSP creates technologies to help NextGen fulfill its promise. AOSP works with the FAA, industry and academic partners to conceive and develop NextGen technologies to improve the intrinsic safety of current and future aircraft. The SASO and SMART-NAS projects are part of AOSP. SASO identifies and develops the maximum possible autonomous capabilities. The SMART-NAS Project will develop an air traffic management simulation capability to explore integration of alternative concepts, technologies and architectures within the National Airspace System. In support of 
SMART-NAS, NASA GRC is working with NASA ARC on Networked ATM architectures and technologies. GRC has a testbed at Cleveland Hopkins Airport to support this effort with emphasis on surface communications.

\section{Goals}

Looking at all the programs that exist to improve safety and efficiency in the global airspace system, one quickly realizes that the keys to success are network-centric operations and improved situational awareness. One of the critical enablers is "connected aircraft". Connectivity to the aircraft must be secure, reliable, and manageable globally.

\section{Modernization Issues}

Why is it so difficult to get new technologies deployed in the global airspace system?

\section{Safety}

The airline industry has an excellent safety record. In order to maintain public confidence in air travel, safety must be the top priority. Thus any new technologies or any changes to operational procedures must be proven to at least maintain the current level of safety if not improve it. This is not an easy matter and can be rather expensive in terms of both time and money. As a result, the industry is often looked at as being reactive rather than proactive.

\section{The "Able" List}

Assuming we can get past the safety issues, then the general problem with deploying new technologies is that they do not meet all the requirements of what we coin the "Able List".

The Able List

- Adaptable

- Affordable

- Deployable

- Evolvable

- Global(able)

- Maintainable

- Manageable

- Reliable

- Scalable

\section{- Securable}

Adaptable: A technology that is not adaptable is not likely to be embraced due to a high probability of obsolescence.

Affordable: If the greatest technology in the world is not affordable, it will not be accepted by the airline industry. Remember, the airlines must make money to survive. This is one reason satellite communications is often not embraced. The cost of the conductivity versus the overall perceived payback is not considered justified by many.

Deployable: If one has to take an aircraft off-line, then the airline cannot generate revenue from that aircraft while the new technology is being integrated. Thus, integration should be done during normal maintenance with little additional out-of-service time.

Evolvable: Technologies must seamlessly integrate into existing systems and any upgrades must be easy to make. There is no flag day ${ }^{1}$.

Global(able): Global scalability must be considered. Airplanes fly from one city to another and from one country to another. All operations must be smooth and seamless and be capable of global deployment - particularly when it comes to security solutions.

Maintainable: One must easily be able to maintain a system once it is deployed.

Manageable: One must be able to easily manage a system in a cost-effective manner.

Reliable: The system must be reliable particularly in the aerospace industry. Reliability affects safety, maintainability and cost.

Scalable: It is significantly more challenging to make systems work on a global scale than it is for small-scale deployment such as confined to an individual airport. Scalability is hard.

Securable: If one cannot secure the system to the scale of its intended deployment it is rendered useless.

\footnotetext{
${ }^{1}$ A change which requires a complete restart or conversion of a sizable body of software or data and requires that updates be performed almost simultaneously across the entire system or network. It came into use when a massive change was made to the Multics time-sharing system to convert from the old ASCII code to the new one; this was scheduled for Flag Day (a US holiday), June 14, 1966.
} 


\section{An Integrated Communication, Navigation and Surveillance System}

Figure 1 illustrates an example of an integrated communication, navigation and surveillance system for Aeronautics. Four major entities are shown here: FAA (or EUROCONTROL or another air traffic management entity depending on where one is in a global deployment), Port Authority, airlines, and PIES. Not shown are UAV. How UAV are handled is a subject for future consideration.
These four entities represent four security domains. Perhaps, with the exception of PIES, each of these entities needs to interact and exchange information in order to provide system-wide situational awareness thereby enabling each entity to properly manage assets and resources with the overall goal of maintaining safety and improving efficiencies.

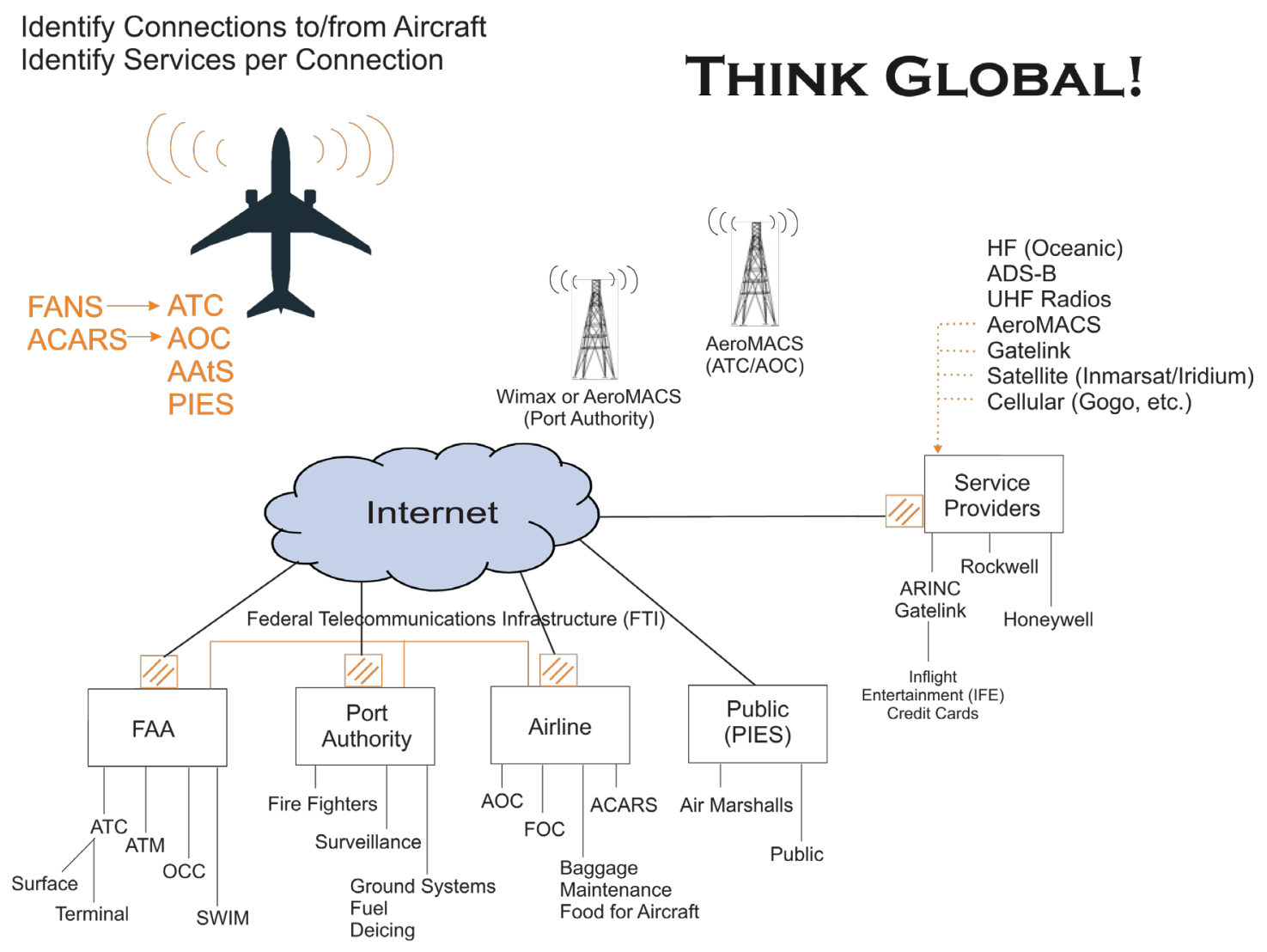

Figure 1. Integrated Communication, Navigation and Surveillance System

A fully connected aircraft communicates over a variety of systems owned and operated by various entities both public and private. These connections include High Frequency (HF) for oceanic, Autromatic Dependent Surveillance - Broadcast (ADS-B), Very High Frequency (VHF) radios, Airport Mobile Access Communication System (AeroMACS), Gatelink, satellite systems such as Inmarsat and Iridium, and cellular systems.
In order to get a sense deployment relative to scalability, consider the AeroMACS deployment for the Port Authority versus AeroMACS deployment for air traffic control in ATC operations. With regard to the Port Authority, the AeroMACS system is entirely controlled by one entity, the Port Authority. Thus, the entire AeroMACS network including all security and QoS configurations are controlled by the Port Authority. The system does not have to scale beyond the airport in question. The AeroMACS AAA server can be local and does not have to share any 
information with any other systems. However, for the ATC AeroMACS deployment, the access into the system must scale to a global level and must handle network layer mobility as a single aircraft may fly throughout the global airspace landing at various airports in different countries. Thus, the aircraft is a system that roams using multiple service providers and wireless technologies. Authorization, access and service agreements ${ }^{2}$ must be manageable and deployable on a global scale. Note, there is a similar need for authorization, access, and service agreements on a global scale for Gatelink, various satellite systems, cellular systems, and future wireless systems.

\section{Globally Deployable, Identity-Based Secure Airspace System}

The remainder of this paper focuses on applying the basic security architectures of commercial telecommunication systems to provide an affordable, scalable, evolvable reliable, maintainable and manageable solution to enable a globally deployable identity-based secure airspace system.

\section{Issues/Requirements}

The following are the system requirements:

1) Seamless Global Access,

2) Seamless Billing,

3) Manageable Service Agreements (e.g. QoS),

4) Scalable Seamless Management,

5) Seamless Roaming, and

6) Single Identity.

In the above requirements, "seamless" implies machine-to-machine communications without human intervention. A single identity is the key to enabling seamless operations.

It is currently unclear how to implement the requirements above in the GAS. Fortunately, the telecommunication industry and in particular the IETF and the 3GPP have developed many standards and architectures to address most of these issues.

\section{What is an Identity?}

In this context, an identity is something that can be used to uniquely identify a system. For aeronautics,

\footnotetext{
${ }^{2}$ Quality of Service settings, often which vary depending on the service provider and wireless technology.
}

that system is an aircraft. Since the identity will be used to gain access into an aeronautical network and for billing, we must be able to authenticate that identity and ensure it is not spoofed. We also desire that an identity is usable across multiple access systems.

In commercial systems there are two basic methods used to identify a system: Certificates or some form of serial number such as an electronic serial number (ESN) or international mobile equipment identity (IMEI). ESNs and IMEIs are used by the cell phone industry. One problem with ESNs and IMEIs is that they are assigned to a specific piece of hardware making portability an issue. A solution to this is the SIM, an integrated circuit that securely stores the International Mobile Subscriber Identity (IMSI) number and its related key which are used to identify and authenticate subscribers on mobile telephony devices. SIM cards are designed to be transferable between different mobile devices. If a piece of equipment breaks or is upgraded, one can simply move the SIM card to the replacement unit and it becomes operational again. No reregistration needs to occur.

An identity certificate or digital certificate is an cryptographic document used to prove ownership of a public key. The certificate includes information about the key, information about its owner's identity, and the digital signature of an entity that has verified the certificate's contents are correct. If the signature is valid, and the entity examining the certificate trusts the signer, then they know they can use that key to communicate with its owner. Identity certificates are associated with the PKI and usually associate with X.509, an ITU-T standard for a public key infrastructure $[1,2]$.

\section{Certificate Management in Aeronautical Systems}

Certificate management is difficult in any system even a single owner system. Regarding aeronautics, in order to be scalable, manageable and affordable, a single identity (a single certificate) is highly desirable. It may be possible to map other certificates to this single identity in order to utilize that single identity across multiple systems owned and operated by different service providers.

In aeronautics systems, various wireless systems have vastly different bandwidth capabilities. 
Likewise, various certificates and keys have vastly different bandwidth requirements. Harmonizing these is a challenge. The FAA sponsored a report on AeroMACS privacy key management [3]. Within this report it was determined that specifying the operation of AAA capabilities was out-of-scope. It was agreed that the MOPS should address the air interface between the subscriber station and the base station with some material to address interoperability with AAA sites (e.g., an interoperable certificate profile). However, Key management and synchronization across all AAA sites was determined to be a ground AeroMACS deployment issue. Some important points from this document follow:

- Key pairs and the corresponding certificates for airborne users are associated with a given airframe, and not, for example, with a pilot or a particular flight identifier. In addition, key pairs and certificates are not assigned to individual pieces of equipment on an airframe.

- ICAO Doc 9880 Part IVB specifies a CA architecture in which each State operates (or contracts with an entity to operate on its behalf) a root-level CA. These State CA have a non-transitive peer relationship amongst one another rather than a hierarchical relationship. The relationship among such $\mathrm{CA}$ is expected to be established and maintained through bilateral and/or multilateral agreements, which includes, for example, provision for cross-certification.

- Harmonization amongst the AeroMACS and ATN based security solutions is to minimize the cryptographic infrastructure implemented on an airframe. Harmonizing these solutions will allow airframe and avionics manufacturers to use common toolsets to provide security services for multiple applications. The areas of harmonization are the underlying cryptographic settings, certificate content, and $\mathrm{CA}$ architecture.

- Provisioning must occur across ATN OSI Security, ATN IPS Security, ARINC 823, WiMAX and AeroMACS

\section{Quality of Service}

Quality of service (QoS) is the overall performance of a service as seen by the users of the network. QoS has numerous characteristics including error rates, bit rate, throughput, transmission delay, availability, and jitter. Different services require different QoS. For example, voice may be able to withstand a high error rate but requires low transmission delay whereas a file transfer may require relatively low error rates but can withstand jitter, transmission delay, and operate over a variety of bit rates. Video may require low error rates, low jitter, and high bit rate. The specification of QoS service parameters is known as a service flow. Per service flow QoS is the ability to identify a traffic flow and enable rules on how that specific flow should be treated including how the flow should behave when forwarded with other traffic flows.

In order to be scalable and manageable, one must be able to specify QoS for a particular entity globally. The QoS specifications are likely to vary per link type (e.g. AeroMAX, Gatelink, Satellite, 4G/5D), but should not vary per service provider for any particular link type. This implies some type of roaming agreements between service providers for any particular link type.

\section{System Access and QoS Provisioning}

In order to understand concepts of system access and QoS provisioning, we will examine two existing systems: Gatelink ${ }^{3}$ and LTE.

\section{System Access}

First, we will summarize the Gatelink access methodology as described in the ARINC specification 822 [4] illustrated in Figure 2. When the aircraft is in range of the airport AP, the aircraft client will scan for available APs. Once the RF association has completed, the aircraft will initiate an authentication session with the airport AP based on the registered aircraft (TWLF) and the AAA server. That server could be at the airport, or at the airline under the Network Administrator's control as shown in figure 6(')@. For global scalability, the AAA server should be at the airline in which case, the AP would also need a RADIUS proxy capability ${ }^{4}$. Proxy service enables a RADIUS/AAA server to forward an authentication request from a local server to a remote RADIUS server and return the remote server's reply to the local

\footnotetext{
${ }^{3}$ We use the Gatelink illustration as Gatelink does not require and QoS management and is therefore a simpler example.

${ }^{4}$ There needs to be a method of securing the proxy transaction between the airport and airline, because the current RADIUS proxy protocol is not secure.
} 
server. In this way, client/server architecture is established between local and remote servers in effect, extending the wireless link from the aircraft to the airline's network.

An X.509 certificate is used to gain access to the system. (It is highly desirable to have one certificate for Gatelink, AeroMACS, WiMAX, SATCOM, LTE, etc. in order to scale globally and be manageable). The X.509 certificate must be signed by a valid certificate authority and must be installed on each Gatelink client (aircraft). The AAA server will acknowledge or deny the signature of the root authority or will forward the authentication requests to the airline or some authorized third party RADIUS server via proxy depending on the agreement with the airline. The remote RADIUS server will acknowledge or deny the request.

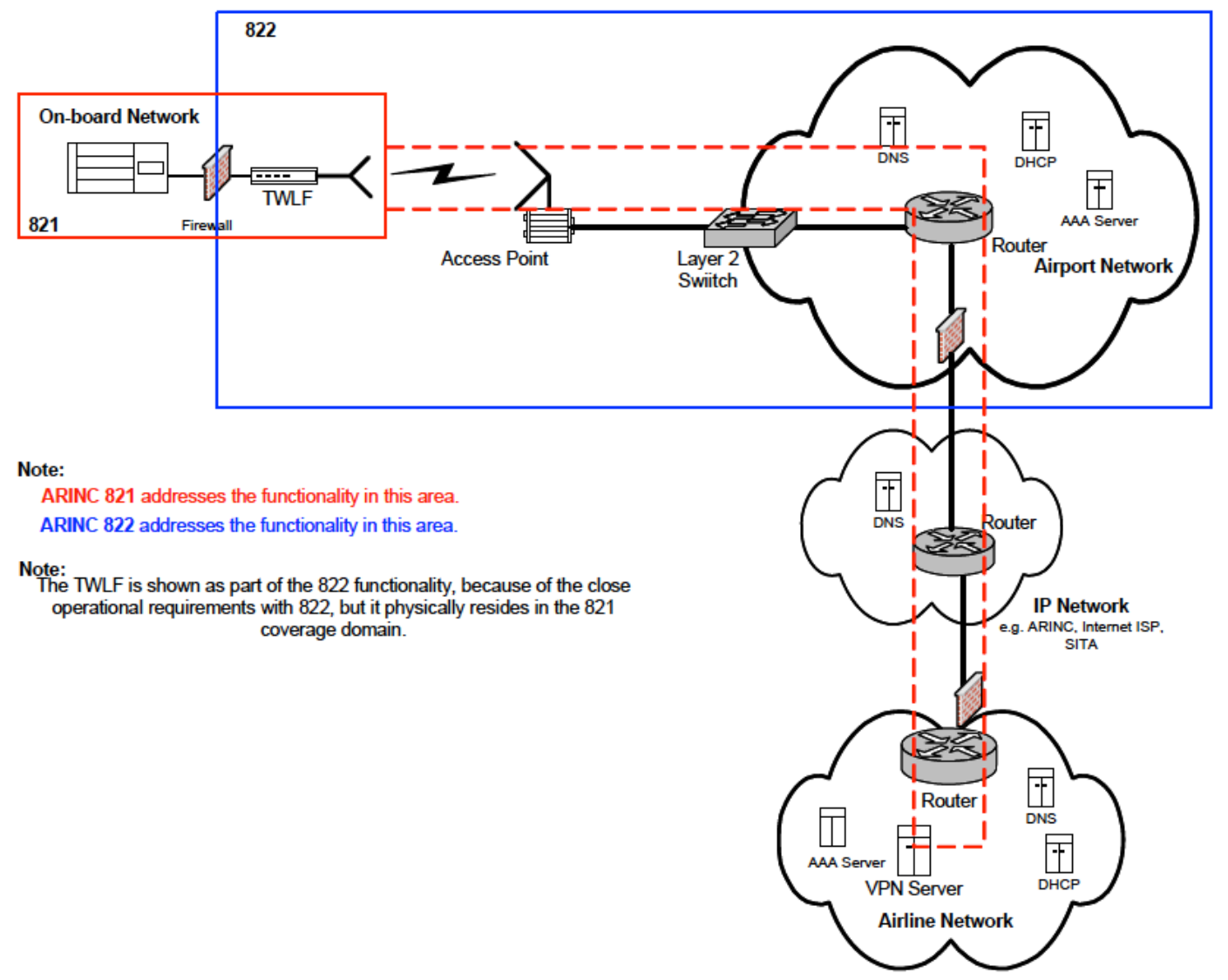

Figure 2. Gatelink Network Architecture Recommendations and Concept of Operations [4]

If the accessing system (or user) is not in the local RADIUS server configurations, the server will look for a realm name and pass the authentication request to a remote RADIUS server which is mapped via manual configuration to the realm name.

The Access System (User-Name) RADIUS attribute is a character string that typically contains the accessing system account's location (called the realm) and the accessing system account's name. The realm is synonymous with the concept of domain, including DNS domains and Active Directory domains. For example, the

URL
http://www.ABCXYZ.com/index.html, the domain name is $A B C X Y Z$.com. which is also the realm name.

IDN may be included in certificates and CRL in the subjectAltName and issuerAltName extensions, name constraints extension, authority information access extension, subject information access extension, CRL distribution points extension, and issuing distribution point extension [5].

Realm names are configured in connection request policies while designing and deploying the RADIUS infrastructure to ensure that connection requests are routed from RADIUS network access 
servers (AAA airport server in figure 6( )@), to remote distributed RADIUS servers (Airline Network AAA server) that can authenticate and authorize the connection request.

Decentralizing AAA has multiple problems that must be considered. These problems mainly relate to user account integrity, user profile management, failover mechanisms, and AAA interworking for roaming users [6]. Other considerations for connected aircraft are acquisition time (the time it takes to get authorization, QoS parameters and IP connectivity to remote AAA systems).

\section{Multi-Domain System Access}

Figure 3 is from a Cisco White paper, Architecture for Mobile Data Offload over Wireless Fidelity (Wi-Fi) Access Networks [7]. This illustrates the relationship between a Third Generation (3G) Radio Access Network ( RAN), an LTE RAN service provider and a cooperating Wi-Fi service provider. When a system wishes to connect to the Wi-Fi RAN, authentication can be setup such that the AAA server at the Wi-Fi Internet Service Provider (ISP) proxies to the 3G/LTE Service Provider (SP) network access system. This allows a non-3GPP Internet Protocol (IP) access into the $3 \mathrm{G}$ MPC. Note, the Policy and Change Control (PCC) is managed by the 3G/LTE SP. The $\mathrm{PCC}$ is where QoS is managed in the $3 \mathrm{GPP}$ network architecture.

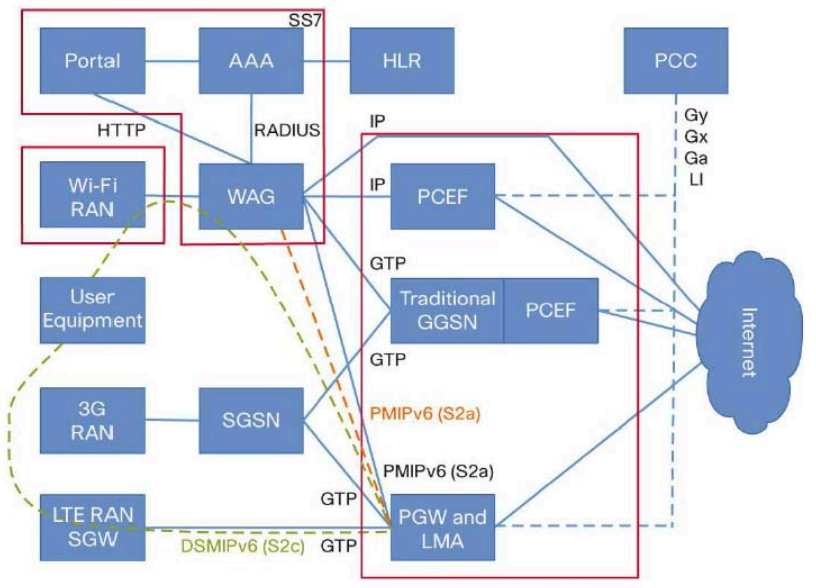

Figure 3. Multi-Domain System Access [7]

To control subscriber access to Wi-Fi networks, multiple authentication methods can be used. In a typical modern Wi-Fi network, two types of authentication are available. The first method, portal based authentication, targets customers without a permanent contract with the operator (vouchers, time-limited access,SMS payments, etc.). Alternatively, EAP authentication provides transparent and easy access for the subscribers with SIM cards or certificates ${ }^{5}$. EAP-based authentication allows transparent authentication and secure communication without interaction from the subscriber (only initial configuration of the SSID which, for Gatelink, is predefined and configured in the TWLF [4].

For an aeronautic global network, visualize the Wi-Fi access as Gatelink, the $3 \mathrm{G}$ as AeroMACS and LTE as a future wireless access.

\section{QoS Provisioning}

For all practical purposes, an aircraft could be considered constantly roaming across multiple wireless networks owned and operated by multiple entities. How one manages QoS over such a diverse network is an interesting challenge. Fortunately, it is not unique to the aeronautics industry. The commercial telecommunication and ISP have had to address this exact problem.

Figure 4, PCC reference architecture for fixed Broadband Access Interworking (visited access), illustrates how QoS and policy is provisioned in a roaming system for a 3 GPP network. Regarding QoS provisioning, the important interface is the $\mathrm{S} 9$ reference point. "S9 resides between a PCRF in the PLMN H-PLMN (H-PCRF) and a PCRF in the V-PLMN (V-PCRF). For roaming with a visited access, this interface reference enables the H-PCRF to:

- Have dynamic PCC control, including the PCEF and, if applicable, BBERF, and, if applicable, TDF, in the V-PLMN;

- Deliver or receive IP CAN specific parameters from both the PCEF and, if applicable, BBERF, in the V-PLMN;

- Serve recieve authorizations and event subscriptions from an AF in the V-PLMN;

- Receive application identifier, service data flow descriptions, if available, application instance identifiers, if available, and application detection start/stop event triggers report.

\footnotetext{
${ }^{5}$ EAP authentication would be use for aeronautics.
} 
For roaming with a home routed access, the S9 interface enables the H-PCRF to provide dynamic
QoS control policies from the H-PLMN, via a V-PCRF, to a BBERF in the V-PLMN [8]."

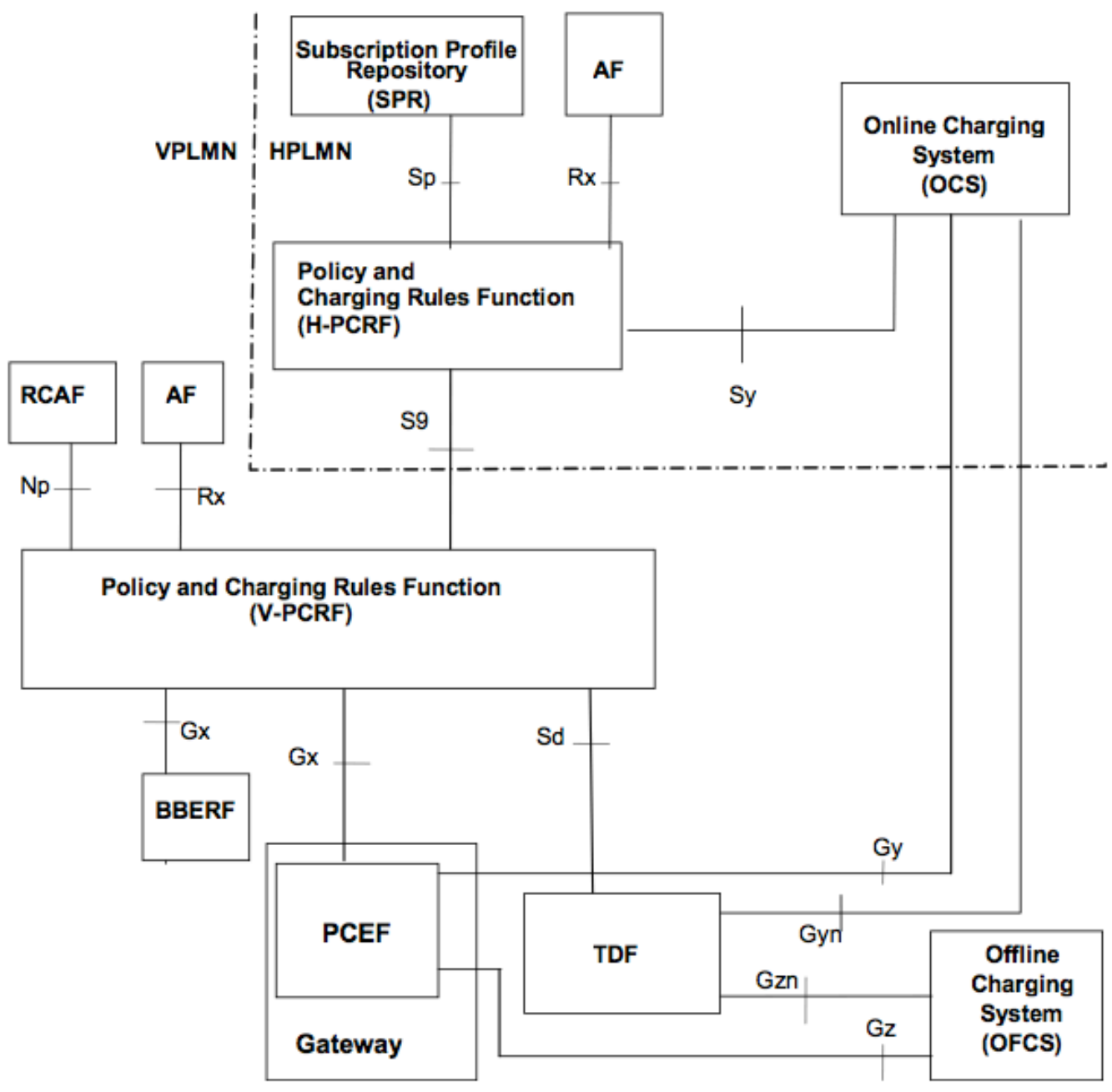

Figure 4. PCC Reference Architecture for Fixed Broadband Access Interworking (Visited Access) [8]

The LTE PCC functions include:

- Policy and Charging Rules Function (PCRF) The PCRF provides policy control and flow based charging control decisions.

- Policy and Charging Enforcement Function (PCEF) The PCEF is implemented in the serving gateway, this enforces gating and QoS for individual IP flows on behalf of the PCRF. It also provides usage measurement to support charging.

- Online Charging System OCS The OCS provides credit management and grants credit to the
PCEF based on time, traffic volume or chargeable events.

- Off-line Charging System OFCS The OFCS receives events from the PCEF and generates CDR for the billing system.

The WiMAX PCC architecture is very similar to $3 \mathrm{GPP}$ as shown in Figure 5. The ASN in WiMAX maps to the RAN in 3GPP while the interface between the $\mathrm{vCSN}$ and hCSN is nearly equivalent to interface reference point S9 in Figure 4. 


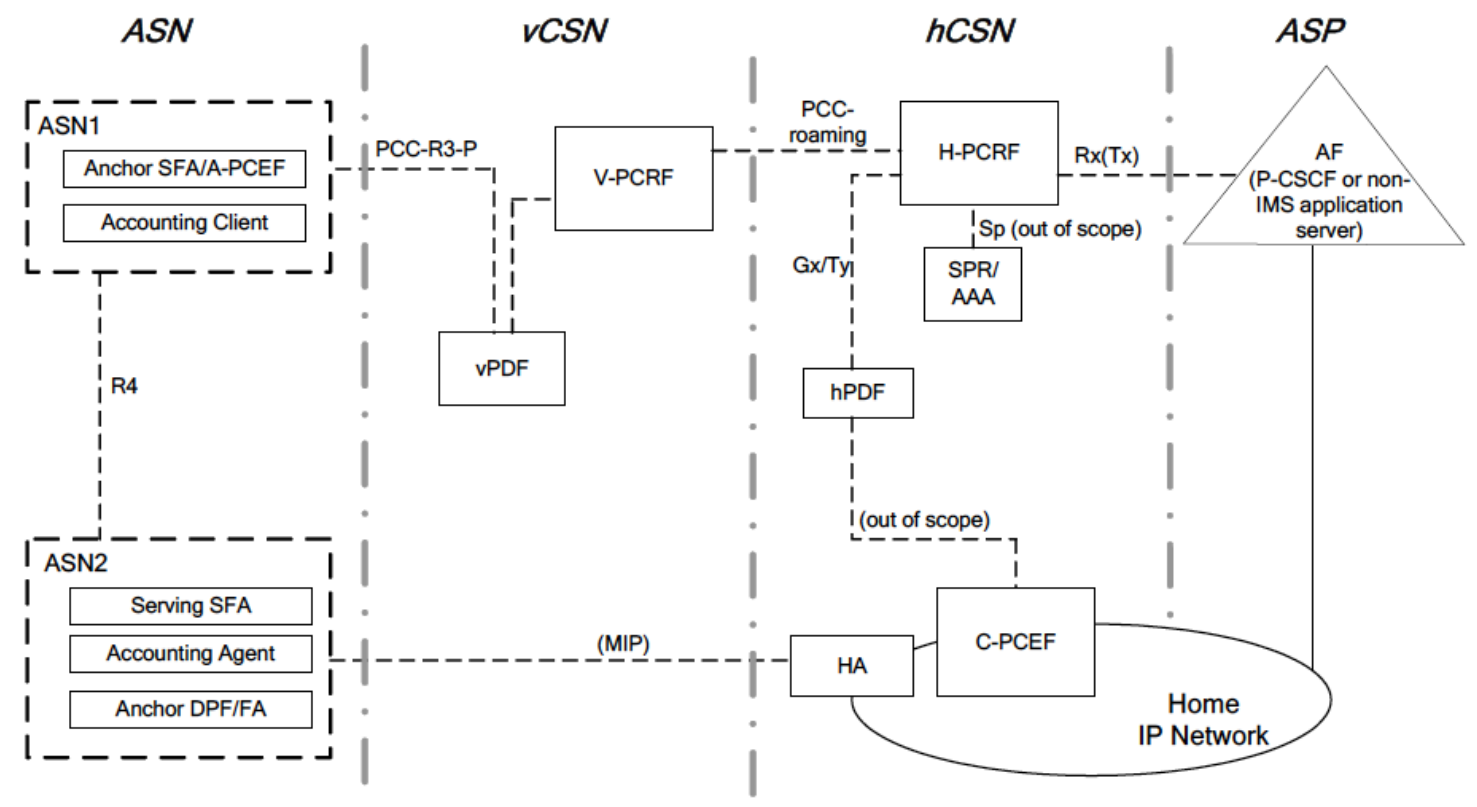

\section{Figure 5. WiMAX Policy Control Architecture - Roaming Scenario with HA in the Home Network [9]}

WiMAX $^{6}$ and 3GPP LTE have been designed with different QoS frameworks and means to enable QoS support for evolving Internet applications. Network initiated or client initiated QoS are both supported in IEEE $802.16 \mathrm{e} / \mathrm{IEEE} 802.16 \mathrm{~m}$ systems. Both operator managed service and unmanaged service can be supported. This flexible architecture gives the mobile client opportunities for differentiation. In contrast, LTE only supports network initiated QoS control [?].

\section{LTE QoS}

3GPP LTE have been designed with a QoS framework to support QoS of evolving Internet applications. LTE offers two types of bearers (classes): GBR and non-GBR. GBR is similar to UGS in WiMAX/AeroMACS. Resources related to a GBR value associated with the bearer are permanently allocated when a bearer becomes established or modified. A non-GBR bearer is the default bearer. A service utilizing a non-GBR bearer may experience congestion-related packet loss [11].

"The QoS level of granularity in the LTE EPS is a packet flow established between the packet data network gateway and the user terminal, the bearer channel. The traffic running between a particular

\footnotetext{
${ }^{6}$ Note, AeroMACS is a tailored instance of WiMAX and uses the same architecture and protocols. As such, AeroMACS implements QoS management in the same manner as WiMAX.
}

client application and a service can be differentiated into separate SDF. SDF mapped to the same bearer channel receive a common QoS treatment (e.g., scheduling policy, queue management policy, rate shaping policy, RLC configuration). Each SDF is associated with one and only one QCI. For the same $I P C A N$ session, multiple SDF with the same QCI and ARP can be treated as a single traffic aggregate which is referred to as an SDF aggregate. Each QCI maps to specific applications. QCI characteristics describe the packet forwarding treatment that an SDF aggregate receives edge-to-edge between the UE and the PCEF in terms of the following performance characteristics:

\section{Resource Type (GBR or Non-GBR);}

2. Priority;

3. Packet Delay Budget;

4. Packet Error Loss Rate [?]."

"The PCC function in LTE networks brings together and enhances capabilities from earlier $3 G P P$ releases to deliver dynamic control of policy and charging on a per subscriber and per IP flow basis. The LTE EPC includes a PCC architecture that provides support for fine-grained $Q o S$ and enables application servers to dynamically control the QoS and charging requirements of the services they deliver. It also provides improved support for roaming. Dynamic control over QoS and charging 
will help operators monetize their LTE investment by providing customers with a variety of $Q o S$ and charging options when choosing a service [?]."

\section{WiMAX QoS}

Five types of scheduling services have been defined for the WiMAX airlink corresponding to the traffic characteristics of different services:

- Unsolicited Grant Service (UGS),

- real-time Polling Service (rtPS),

•non- real-time Polling Service (nrtPS),

- extended-real-time Polling Service (ertPS) and

- Best Effort (BE).

UGS, rtPS and ertPS are mainly used for real-time traffic and interactive traffic such as VoIP, video and online gaming, while nrtPS and $\mathrm{BE}$ are usually utilized for non-real-time traffic such as file transfers, emails, and web browsing.

Each service class has a myriad of QoS parameters associated with uplink/downlink scheduling for a service flow (e.g. Maximum sustained rate, Maximum reserved traffic rate, Maximum latency, Jitter tolerance, Packet loss, throughput) [13].
Traffic classification and mapping from application packets onto SF in WiMAX is done at the CS. Classification is often done using a five-tuple, such as source and destination IP addresses, source and destination port address, protocol, and DSCP [4].

During the creation of a SF, the CS that the connection will use is defined. Possible choices of CS are No CS, Packet IPv4, Packet IPv6, Packet 802.3/Ethernet, Packet 802.1Q VLAN ${ }^{7}$, Packet IPv4 over 802.3/Ethernet, Packet IPv6 over 802.3/ Ethernet, Packet IPv4 over 802.1Q VLAN, Packet IPv6 over 802.1Q VLAN and Asynchronous Transfer Mode [10].

\section{Initial Network Access Example for WiMAX}

To clarify and simplify understanding of network access, a simple WiMAX example is provided. Figure 6 shows the reference architecture for providing service to a roaming Mobile Station (MS) with usage of the Home Agent (HA) in the visited CSN. Authentication, authorization as well as policy information (the QoS profile) is provided from the home CSN to the visited CSN over the reference point R5. Accounting information is forwarded from the visited CSN to the home CSN over R5, and access to services in the home CSN may also be provided over R5 whereas Internet access is usually established directly out of the visited CSN. Note, it is possible and a valid configuration to be roaming with the HA located in the home Network Service Provider (NSP) [15].

\footnotetext{
${ }^{7}$ IEEE 802.1Q standard defines a system of tagging for Ethernet frames and the accompanying procedures to be used by bridges and switches in handling such frames. The standard also contains provisions for a quality of service prioritization scheme.
} 


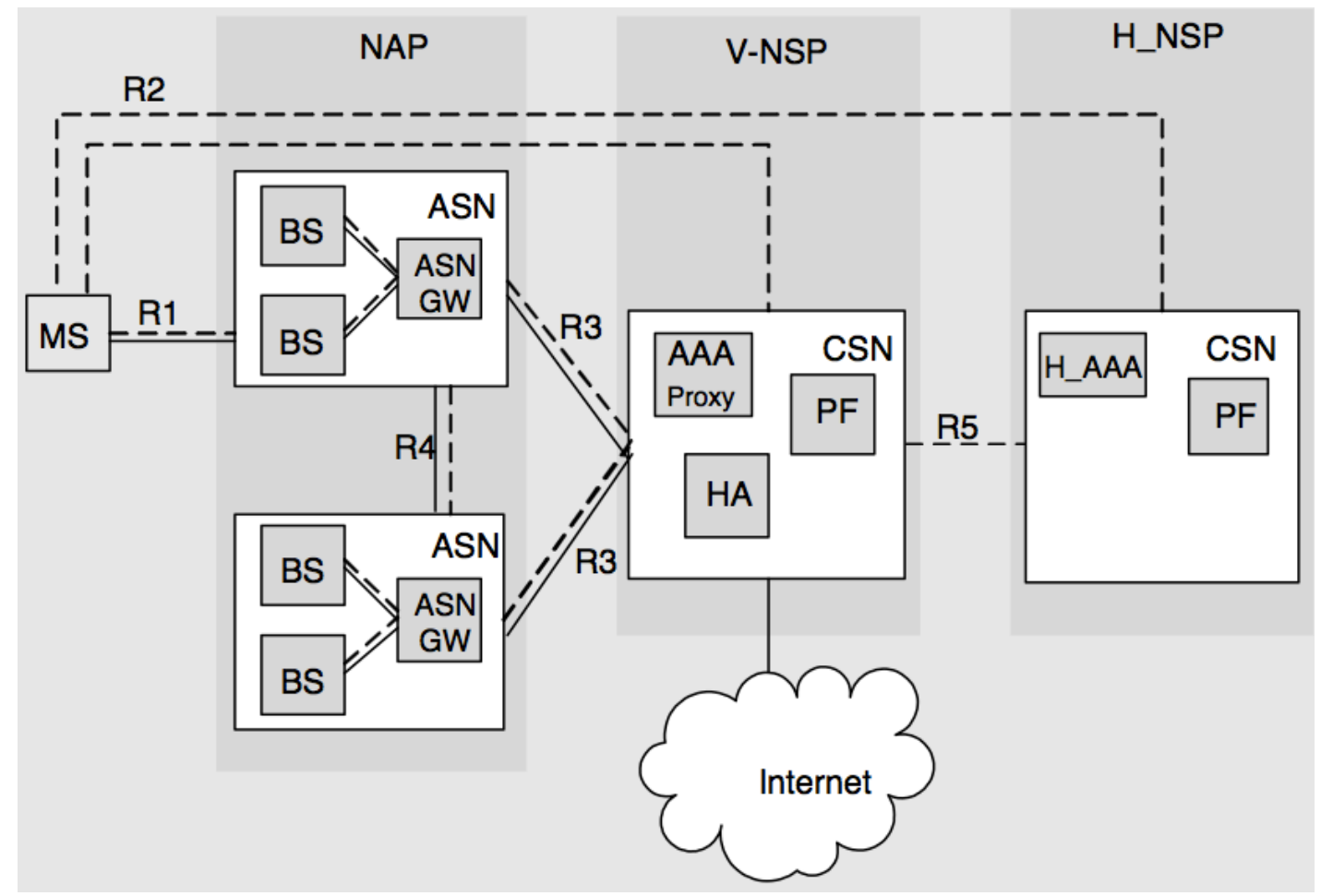

Figure 6. WiMAX Roaming with HA Located in the Visited NSP [15]

\section{Authentication, Authorization, and Accounting (AAA) Servers}

The AAA server, QoS management, the PF and PCC are key elements of any of the modern telecommunication architectures. These functions are often performed using a RADIUS [16] or Diameter [17] server.

The RADIUS protocol carries authentication, authorization and configuration information between a NAS and a RADIUS authentication server. Requests and responses carried by the RADIUS protocol are called RADIUS attributes. These attributes can be username, Service-Type, and so on. These attributes provide the information needed by a RADIUS server to authenticate users and to establish authorized network service for them. The RADIUS protocol also carries accounting information between a NAS and a RADIUS accounting server [18].

The RADIUS-Based Policing feature enables the PCEF in the access network to make automatic changes to the policing rate of specific sessions and services. Policies can be based on any attribute in a request or response, include checking both the existence of (or lack of) an attribute, and the contents of an attribute. They can filter out attributes, or re-write the contents of attributes. Attributes can be created, deleted, or edited in a policy. Policies can leverage information in SQL, LDAP, flat-text files, or any other source of data. Policies can be based on identities (user, group, or role), location (client IP, port, etc.), time (date, time of day), authentication method (PAP, CHAP, EAP type, etc.), or any other piece of information that is in a RADIUS packet or in a database. Policies can enforce VLAN capabilities, filtering QoS, etc. [19].

AAA protocols such as RADIUS were initially deployed to provide dial-up PPP and terminal server access. Over time, AAA support was needed on many new access technologies as the scale and complexity of AAA networks grew. AAA was also used on new applications (such as VoIP). This led to new demands on AAA protocols. As a result, Diameter ${ }^{8}$ was developed - evolving from and replacing the much less capable RADIUS protocol. Diameter is not directly backwards compatible but provides an upgrade path for RADIUS.

\footnotetext{
${ }^{8}$ The name is a play on words, derived from the RADIUS protocol. A diameter is twice the radius.
} 
The new network access requirements for AAA protocols addressed by Diameter are summarized below.

- Failover

- Transmission-level security - RADIUS support for IPsec is not required.

- Reliable transport - RADIUS runs over UDP, and does not define retransmission behavior; as a result, reliability varies between implementations.

- Agent support - RADIUS does not provide for explicit support for agents, including proxies, redirects, and relays. Since the expected behavior is not defined, it varies between implementations.

- Server-initiated messages

- Transition support - Considerable effort has been expended in enabling backward compatibility with RADIUS so that the two protocols may be deployed in the same network.

- Capability negotiation

- Peer discovery and configuration - RADIUS implementations typically require that the name or address of servers or clients be manually configured, along with the corresponding shared secrets. This results in a large administrative burden and creates the temptation to reuse the RADIUS shared secret, which can result in major security vulnerabilities if the Request Authenticator is not globally and temporally unique as required in RFC2665 [?].

Over time, the capabilities of Network Access Server (NAS) devices have increased substantially. As a result, Diameter is a considerably more sophisticated protocol than RADIUS Through the use of extensions, the protocol was designed to be extensible to support proxies, brokers, strong security, mobile IP, network-access servers, accounting and resource management.

\section{Summary}

In order to globally deploy new communications technologies into the GAS those technologies must meet all the requirements of the "Able List". The GAS currently consists of a variety of communications links, often quite old such as VHF analog radios with limited bandwidth capability. New technologies such as Gatelink and AeroMACS offer greater capability, greater bandwidth, better security and potential cost savings. However, these systems will not be deployed if the cost of deployment and management outweighs the benefits. Identity-based security with single certificate sign-on for system access along with the capability of managing QoS policy for diverse systems in a centralized location has the potential to ensure a smooth, evolvable, scalable, manageable, affordable deployment. Modern telecommunications networks have shown this to be possible for single communication technologies types (e.g. LTE and WiMAX). In addition the tools, protocols and architectures exist. The outstanding question remains: "Can a single identity and centralized QoS policy management be deployed that encompasses multiple ASNs and NSPs to enable connected aircraft?"

\section{References}

[1] I. Recommendation, "509 (2005)| iso/iec 95948: 2005," Information Technology-Open Systems Interconnection-The Directory: Public-Key and at Tribute Certificate Frameworks,

[2] C. Adams, S. Farrell, T. Kause, and T. Mononen, "Internet x.509 Public Key Infrastructure Cerficate Management Protocol (cmp)," Internet Engineering Task Force, RFC 4210, Sep. 2005. [Online]. Available: http://www.rfc-editor.org/rfc/rfe4210.txt.

[3] I. Exelis, "New ATM Requirements - Future Com-Munications, C-Band and L-Band Communications Study Aeromacs Privacy Key Management Report," FAA, Final Report SE2020 TO $0008 \quad$ (TORP 1240) Task 3.2 Contract DTFAWA-10-D-00028, 2012.

[4] "Aircraft / Ground Aircraft / Ground Ip Communication (Gatelink)," ARINC, Tech. Rep. ARINC SPEC-IFICATION 822-1, 2008.

[5] D. Cooper, S. Santesson, S. Farrell, S. Boeyen, Housley, and W. Polk, "Internet x.509 Public Key Infrastructure Certificate and Certificate Revocation List (CRL) Profile," Internet Engineering Task Force, RFC 5280, May 2008. [Online]. Available: http://www.rfc-editor.org/rfc/rfc5280.txt.

[6] D. Forsberg, "Secure Distributed AAA with Domain and User Reputation," in World of Wireless, Mobile and Multimedia Networks, 2007. WoWMoM 2007. IEEE International Symposium on a, IEEE, 2007, pp. 1-6. 
[7] "Architecture for Mobile Data Offload Over Wi-fi Access Networks," Cisco System, White Paper C11-701018-00, 2012. [Online]. Available: http://tools.

cisco.com/search/results/display?url=http $\% 3 \mathrm{a} \% 2 \mathrm{f} \% 2$ fwww.cisco.com $\% 2 \mathrm{fc} \% 2 \mathrm{fen} \% 2 \mathrm{fus} \% 2$ fsolutions $\% 2 \mathrm{fc}$ ollateral $\% 2$ fservice-provider $\% 2$ fservice-providerwifi\%2fwhite paper_c11-701018.pdf\&pos $=1 \&$ query $=\mathrm{C}$ 11-701018-00.

[8] 3GPP, "Policy and Charging Control Architecture," 3GPP, Technical Spefication 3GPP

TS 23.203, 2015. [Online]. Available: http://www.3gpp.org/ftp/Specs/archive/23_series/23. 203/23203-d40.zip.

[9] "Architecture, Detailed Protocols and Procedures Policy and Charging Control," WiMAX Forum, Tech. Rep. WMF-T33-109-R015v02, 2010.

[10] M. Alasti, B. Neekzad, J. Hui, and R. Van-nithamby, "Quality of Service in Wimax and Lte Net-Works [Topics in Wireless Communications]," Communications Magazine, IEEE, vol. 48, no. 5, pp. 104- 111, 2010.

[11] H. Ekström, "QoS Control in the 3GPP Evolved Packet System," Communications Magazine, IEEE, vol. 47, no. 2, pp. 76-83, 2009.

[12] 2015. [Online]. Available: http://lteworld.org/ ltefaq/how-does-policy-control-and-charging-workslt e.

[13] Wimax technology for broadband wireless access -7.4, August. [Online]. Available: http://etutorials.org/Networking/wimax + technology + broadband + wireless + access $/$ Part + Three + WiMAX + Multiple + Access + MAC + Layer + and + Qos + Management / Chapter $+7+$ Convergence + Sublayer+CS/7 . 4+CS+ and +QoS/.

[14] F. Baker, J. Polk, and M. Dolly, "A DifferentiAted Services Code Point (DSCP) for Capacity-Admitted Truff," Internet Engineering Task Force, RFC 5865, May 2010. [Online]. Available: http://www.rfc-editor. org/rfc/rfc5865.txt.

[15] "Stage 2: Architecture Tenets, Reference Model and Reference Points," WiMAX Forum, Tech. Rep. WMF-T32-002-R010v04, 2009.

[16] C. Rigney, S. Willens, A. Rubens, and W. Simp-son, "Remote Authentication Dial in User Service (RADIUS)," Internet Engineering Task Force,

RFC 2865, Jun. 2000. [Online]. Available: http://www.rfc-editor.org/rfc/rfc2865.txt.

[17] V. Fajardo, J. Arkko, J. Loughney, and G. Zorn, "Diameter Base Protocol," Internet Engineering Task Force, RFC 6733, Oct. 2012. [Online]. Available: http://www.rfc-editor.org/rfc/rfc6733.txt.

[18] Cofiguring Radius -Based Policing, August. [On-line]. Available:

http://www.cisco.com/c/en/us/td/

docs/ios-xml/ios/isg/configuration/xe-3s/isg-xe-3s-bo ok/isg-radius-pol.html\#GUID-1CDFAA27-177E-42F 7-AD9F-05A17248526D.

[19] 2015. [Online]. Available: http://freeradius.org/ features/policy.html.

\section{Acronyms List}

3G

3GPP

AAA

ADS-B

AeroMAC

AOSP

ARINC

ARP

ASN

ATC

ATM

ATN

AP

BBERF

BE

CA

CAN
Third Generation

3rd Generation Partnership Project

Authentication, Authorization and Accounting

Automatic Dependent Surveillance -Broadcast

SAirport Mobile Access Communication System AF Application Function ARC Ames Research Center Airspace Operations and Safety Program

Aeronautical Radio, Incorporated Allocation and Retention Priority Access Service Network Air Traffic Control

Air Traffic Management

Aeronautical

Telecommunication Network Access Point

Bearer Binding and Event Reporting Function

Best Effort

Certificate Authority Challenge-Handshake Authentication Protocol Connectivity Access Network 


\begin{tabular}{|c|c|c|c|}
\hline CDR & Charging Data Records & MPC & Mobile Packet Core \\
\hline \multirow[t]{2}{*}{ CHAP } & Challenge-Handshake & MS & Mobile Station \\
\hline & Authentication Protocol & NAS & Network Access Server \\
\hline CRL & Certificate Revocation List & NASA & National Aeronautics and Space \\
\hline CS & Convergence Sublayer & NextGen & Administration Next Generation \\
\hline CSN & Connection Service Network & & Air Transportation System \\
\hline DNS & Domain Name System & nrtPS & non-real-time Polling Service \\
\hline \multirow[t]{2}{*}{ DSCP } & Differentiated Services Code & NSP & Network Service Provider \\
\hline & Point & OCS & Online Charging System \\
\hline \multirow[t]{2}{*}{ EAP } & Extensible Authentication & OFCS & Off-line Charging System \\
\hline & Protocol & OSI & Open System Interconnection \\
\hline EPC & Evolved Packet Core & PAP & Password Authentication \\
\hline EPS & Evolved Packet System & & Protocol \\
\hline ertPS & extended-real-time Polling & PCC & Policy and Change Control \\
\hline EUROCONTROL & $\begin{array}{l}\text { Service } \\
\text { European Organisation for the } \\
\text { Safety of Air Navigation }\end{array}$ & PCEF & $\begin{array}{l}\text { Policy and Charging } \\
\text { Enforcement Function }\end{array}$ \\
\hline FAA & $\begin{array}{l}\text { Satety of Alr Navigation } \\
\text { Federal Aviation Administration }\end{array}$ & PCRF & Policy and Charging Rules \\
\hline GAS & Global Airspace System & PF & $\begin{array}{l}\text { Function } \\
\text { Policy Function }\end{array}$ \\
\hline GBR & Guaranteed Bit Rate & PIES & Public Internet and \\
\hline GPS & Global Positioning System & & Entertainment Services \\
\hline GRC & Glenn Research Center & PKI & Public Key Infrastructure \\
\hline HA & Home Agen & PLMN & Public Land Mobile Network \\
\hline hCSN & Home CSN & PPP & Point-to-Point Protocol \\
\hline HF & High Frequency & QCI & QoS Class Identifier \\
\hline H-PCRF & Home PCRF & QoS & Quality-of-Service \\
\hline H-PLMN & Home PLMN & RADIUS & Remote Authentication Dial-In \\
\hline \multirow{2}{*}{ IATA } & International Air Transport & & User Service \\
\hline & Association & RAN & Radio Access Network \\
\hline \multirow[t]{2}{*}{ ICAO } & International Civil Aviation & $\mathbf{R F}$ & Radio Frequency \\
\hline & Organization & RLC & Radio Link Control \\
\hline IDN & Internationalized Domain Name & rtPS & real-time Polling Service \\
\hline IETF & Internet Engineering Task Force & SASO & Safe Autonomous Systems \\
\hline IPS & Internet Protocol Suite & & Operations \\
\hline IP & Internet Protocol & SATCOM & Satellite Communications \\
\hline ISP & Internet Service Provider & SDF & Service Data Flow \\
\hline \multirow[t]{2}{*}{ ITU-T } & International Telegraph Union & SESAR & Single European Sky ATM \\
\hline & $\begin{array}{l}\text {-Telecommunication } \\
\text { standardization sector }\end{array}$ & SF & $\begin{array}{l}\text { Research } \\
\text { Service Flow }\end{array}$ \\
\hline \multirow[t]{2}{*}{ LDAP } & Lightweight Directory Access & SIM & Subscriber Identification Module \\
\hline & Protocol & SMART-NAS & Shadow Mode Assessment Usir \\
\hline LTE & Long Term Evolution & & Realistic Technologies for the \\
\hline \multirow[t]{2}{*}{ MOPS } & Minimum Operational & & National Airspace System \\
\hline & Performance Specification & SMS & Short Message Service \\
\hline
\end{tabular}




$\begin{array}{ll}\text { SP } & \text { Service Provider } \\ \text { SQL } & \text { Structured Query Language } \\ \text { SSID } & \text { Service Set ID } \\ \text { TDF } & \text { Traffic Detection Function } \\ \text { TWLF } & \text { Terminal Wireless LAN Functions } \\ \text { UAV } & \text { Unmanned Air Vehicle } \\ \text { UE } & \text { User Equipment } \\ \text { UGS } & \text { Unsolicited Grant Service } \\ \text { VCSN } & \text { Visiting CSN } \\ \text { VLAN } & \text { Virtual Local Area Network } \\ \text { V-PCRF } & \text { Visiting PCRF }\end{array}$

$\begin{array}{ll}\text { V-PLMN } & \text { Visiting PLMN } \\ \text { VHF } & \text { Very High Frequency } \\ \text { VoIP } & \text { Voice-Over-IP } \\ \text { Wi-Fi } & \text { Wireless Fidelity } \\ \text { WiMAX } & \text { Worldwide Interoperability for } \\ & \text { Microwave Access }\end{array}$

34th Digital Avionics Systems Conference September 13-17, 2015 